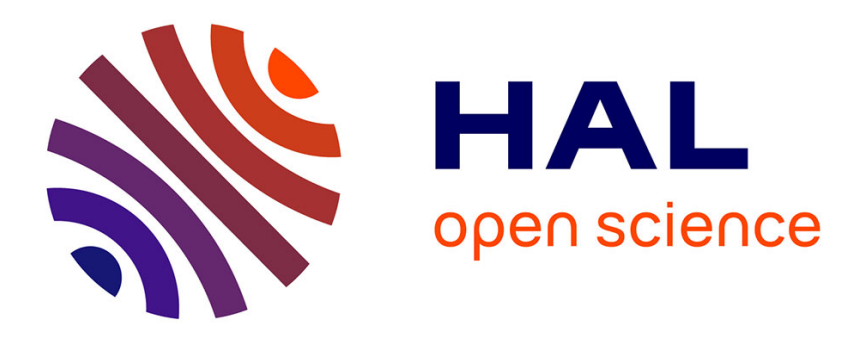

\title{
Study of the deformation of ferrofluid droplets in a magnetic field
}

\author{
J.-C. Bacri, D. Salin, R. Massart
}

\section{To cite this version:}

J.-C. Bacri, D. Salin, R. Massart. Study of the deformation of ferrofluid droplets in a magnetic field. Journal de Physique Lettres, 1982, 43 (6), pp.179-184. 10.1051/jphyslet:01982004306017900 . jpa-00232028

\section{HAL Id: jpa-00232028 https://hal.science/jpa-00232028}

Submitted on 1 Jan 1982

HAL is a multi-disciplinary open access archive for the deposit and dissemination of scientific research documents, whether they are published or not. The documents may come from teaching and research institutions in France or abroad, or from public or private research centers.
L'archive ouverte pluridisciplinaire HAL, est destinée au dépôt et à la diffusion de documents scientifiques de niveau recherche, publiés ou non, émanant des établissements d'enseignement et de recherche français ou étrangers, des laboratoires publics ou privés. 
Classification

Physics Abstracts

$68.10-75.50 \mathrm{~B}-75.50 \mathrm{M}$

\title{
Study of the deformation of ferrofluid droplets in a magnetic field
}

\author{
J.-C. Bacri, D. Salin \\ Laboratoire d'Ultrasons (*), Université Pierre et Marie Curie, \\ Tour 13, 4, place Jussieu, 75230 Paris Cedex 05, France \\ and R. Massart \\ Laboratoire de Chimie des Métaux de Transition (*), Université Pierre et Marie Curie, \\ Tour 54, 4, place Jussieu, 75230 Paris Cedex 05, France
}

(Reçu le 18 novembre 1981, accepté le 29 janvier 1982)

\begin{abstract}
Résumé. - Nous avons étudié dans un nouveau type de ferrofluide la déformation de gros agglomérats ferromagnétiques ( 2 à $20 \mu \mathrm{m}$ ) dans un faible champ magnétique (jusqu'à 10 gauss). La forme ellipsoïdale des agglomérats est due à la compétition entre les forces magnétiques qui tendent à aligner les agrégats dans la direction du champ et la tension de surface qui favorise la forme sphérique. Nous déduisons de nos mesures une valeur de la tension interfaciale des agrégats
\end{abstract}

$$
\sigma=(1,5 \pm 0,2) \cdot 10^{-4} \mathrm{erg} \cdot \mathrm{cm}^{-2} .
$$

\begin{abstract}
We have studied the deformation of large magnetic agglomerates ( 2 to $20 \mu \mathrm{m}$ ) in a weak magnetic field (up to 10 gauss) for a new kind of ferrofluid. The ellipsoid shape of the agglomerate is due to a competition between magnetic energy which favours elongated agglomerates in the field direction and surface energy which favours a spherical shape. We can deduce a value of the interfacial tension of the agglomerates $\sigma=(1.5 \pm 0.2) \cdot 10^{-4} \mathrm{erg} . \mathrm{cm}^{-2}$.
\end{abstract}

1. Introduction. - The most studied ferrofluids are colloidal suspensions of ferromagnetic grains. Typical sizes of nearly spherical grains are of the order of $100 \AA$. Stabilization of the suspension is usually obtained by coating the grains with a polymeric surfactant. In such a kind of ferrofluid [1, 2] agglomeration phenomena occur in the presence of a magnetic field. They have been studied by optical transmission measurements [3-8] and settling velocity measurements [9-11]. P. G. de Gennes and P. A. Pincus [12] have predicted chain formation in dilute ferrofluids, but the observed agglomerates are much thicker than the predictions. More recently Liao and Krueger [13] developed a theory of the formation of agglomerates under magnetic fields. We report here on direct microscope measurements of the size and shape of large agglomerates $(2-20 \mu \mathrm{m})$ under weak magnetic field (from 0 gauss to 10 gauss) in a new kind of ferrofluid [14, 15].

(*) Associated with the Centre National de la Recherche Scientifique. 
2. Theory. - Liao and Krueger [13] have studied the settling agglomeration in a magnetic field. They predict the volume (i.e. the number of particles $N$ ) and the shape of agglomerates in an increasing magnetic field. All agglomerates must have the same volume and shape for a given magnetic field. In our experiment we start, in zero magnetic field, with spherical aggregates of different sizes ( 2 to $20 \mu \mathrm{m}$ diameter) and we follow their size and shape in an increasing magnetic field. The basic idea is that the equilibrium shape of an agglomerate of $N$ grains in a magnetic field $H$ is the result of the competition between the magnetic energy $E_{\mathrm{M}}$ of the agglomerate and its surface energy $E_{\mathrm{S}}$ : magnetic energy favours elongated agglomerates in the direction of the field, whereas surface tension favours the sphere. Calculation of the magnetic energy $E_{M}$ of interaction of the dipoles in an agglomerate in an effective local magnetic field is only possible in special cases such as an ellipsoidal agglomerate [16]. It is this shape we observe. Moreover, as was first pointed out in reference [12], the stability depends on whether we have a prolate (cigar) or an oblate (disk) ellipsoid of revolution. For ferromagnetic grains a prolate ellipsoidal configuration along the magnetic field is appropriate.

The agglomerate contains $N$ nearly spherical grains of radius $R$ at a mean distance $d$. Each grain behaves as a dipole of moment, $\mu=4 \pi R^{3} M / 3$ where $M$ is the magnetization of the ferromagnetic substance of the grains $(M \sim 400 \mathrm{G}$ for magnetite). For an assembly of $N$ permanent dipoles, in a cubic array, at temperature $T$ and in a magnetic field $H$, the magnetic energy $E_{\mathrm{M}}$ is

$$
E_{\mathrm{M}}=-\frac{N \mu^{2}}{2 d^{3}} \begin{cases}Z+h^{2} / h_{\mathrm{c}} & \text { for } h<h_{\mathrm{c}} \\ Z+2 h-h_{\mathrm{c}} & \text { for } h>h_{\mathrm{c}}\end{cases}
$$

$h=H d^{3} / \mu$ is the dimensionless magnetic field; $h_{\mathrm{c}}$ is the field above which the magnetization of the dipolar array is a constant (saturation) :

$$
h_{\mathrm{c}}=Z+l-\frac{4 \pi}{3}
$$

$l$ is the shape dependent demagnetization coefficient $[13,17](l=4 \pi / 3$ for a sphere $) ; Z$ depends on the temperature and on the structure of the cubic array; at $T=0$ [16] $Z=Z_{0}$ equals 5.352 for a simple cubic crystal, $Z_{0}=3.972$ for a face centred one and $Z_{0}=3.616$ for a body centre cubic. Since the energy per dipole is smallest in the simple cubic crystal, we assumed that agglomerates have nearly this structure. Anyway as $T \neq 0$ in our experiment, $Z=Z(T)$ [18] would act as a free parameter to fit the data.

The surface energy $E_{\mathrm{S}}$ of the agglomerate of surface $S$ is

$$
E_{\mathrm{S}}=\sigma S
$$

where $\sigma$ is the unknown surface tension of the aggregate in the ferrofluid. For a prolate ellipsoid,

$$
S=2 \pi a^{2} K\left[K+\varepsilon^{-1} \sin ^{-1} \varepsilon\right] .
$$

The aspect ratio $K=b / a$ is the ratio of the semi-minor $b$ to the semi-major $a$ axis of the ellipsoid; $\varepsilon=\sqrt{1-K^{2}}$.

The size, i.e. the volume $V$ of the agglomerate is given through the number $N$ of dipoles $N=V / d^{3}$. The four variables of our problem are $H, N, V$ and $K$. Since our agglomerates are unstable, but with a life time much longer than our experiment duration, we cannot get any theoretical relation for $V$. We have then to measure it in our experiment. Therefore we only minimize the energy with respect to the aspect ratio $K$ for a given $H, V$ and $N$ :

$$
\frac{\partial}{\partial K}\left(E_{\mathrm{S}}+E_{\mathrm{M}}\right)=0
$$


for $H<H_{\mathrm{c}}\left(H_{\mathrm{c}}=h_{\mathrm{c}} \frac{\mu}{d^{3}} \gtrsim 100\right.$ gauss $)$. Equation (5) gives

$$
H^{2} V^{1 / 3}=\sigma f(K)
$$

with

$$
\begin{aligned}
f(K) & =(\pi / 6 K)^{1 / 3} \varepsilon^{-2}[Z+l-4 \pi / 3]^{2}\left[1+2 K^{2}+\left(1-4 K^{2}\right) K^{-1} \varepsilon^{-1} \sin ^{-1} \varepsilon\right] \frac{\partial K}{\partial l} \\
l & =2 \pi K^{2} \varepsilon^{-3}(-2 \varepsilon+\ln [(1+\varepsilon) /(1-\varepsilon)]) .
\end{aligned}
$$

Then for any agglomerate the cube root of the volume times the square of the magnetic field is a function of the aspect ratio $K$ with only one structure parameter $(Z)$.

3. Experimental set-up. - We use a ferrofluid obtained through a chemical process by the Massart method $[14,15]$. The ferrofluid preparation is carried out in an aqueous solution without surfactant and involves the precipitation of colloidal magnetite. In an alkaline medium the ferrofluid is a negative colloidal solution (macroanion) stabilized by a low polarizing cation [14, 15]. With the molar concentration in ionic iron of our ferrofluid and with the size of each grain $2 R \sim 100 \AA$, the mean distance between grains is nearly $200 \AA$. Without ultracentrifugalization and under a $1000 \mathrm{G}$ magnetic field, two phases appear (one more concentrated than the other one). When the magnetic field is turned off, we obtain a dispersion of droplets ( 2 to $20 \mu \mathrm{m})$ of the concentrated phase in the other. These nearly spherical droplets are metastable and persist a few days for the bigger droplets (Picture 1).

A ferrofluid sample is put in an optical glass tank $4 \mathrm{~cm} \times 0.5 \mathrm{~cm}$ and $200 \mu \mathrm{m}$ thick. The $200 \mu \mathrm{m}$ thickness is in the vertical direction. We observe our sample in this direction through a microscope with a 200 magnifying power. Our length measurement accuracy is then of the order of $1 \mu \mathrm{m}$. The applied magnetic field is horizontal in the larger direction of our sample $(4 \mathrm{~cm}) . H$ is uniform over our volume of observation.

As the magnetic field $H$ is increased we observe (Pictures 2, 3, 4) :

i) formation of prolate ellipsoids in the direction of $H$;

ii) for a given agglomerate, the deformation increases with the field;

iii) for a given $H$, the deformation increases with the size of the agglomerate;

iiii) some agglomerates collapse : agglomerates 4 and 8 (Picture 2) collapse in agglomerate 4 (Picture 3); then agglomerate 4 collapses with 5 to give agglomerate 4 in picture 4 .

We have verified with a vertical magnetic field that the shape of the agglomerate is symmetric to revolution around the direction of $H$. Therefore we analyse the evolution of the shape of agglomerates using prolate ellipsoids.

We have increased the magnetic field step by step up to 10 gauss. For each value of $H$ we wait a few minutes until the agglomerate stabilizes its shape and then we take a picture. In the magnetic field range 0-10 gauss we can follow the evolution of nine different agglomerates. For each agglomerate and each value of the field, we measure the value $a$ and $b$ of the semi-major and semiminor axis of the ellipsoid. Then we compute the volume $V=4 \pi a b^{2} / 3$ and aspect ratio $K=b / a$. As $H$ increases, the volume of each agglomerate decreases up to a factor of 2 . This decrease in volume might be understood as a magnetostriction effect [17] which ejects some water of the agglomerate.

In order to test theoretical predictions (formula (6)), we plot, on a Log-Log scale (Fig. 1) the product $H^{2} V^{1 / 3}$ versus the aspect ratio $K$ for our nine agglomerates and for different magnetic fields. As is clearly shown in the figure, the product $H^{2} V^{1 / 3}$ is a universal function of $K$ over 


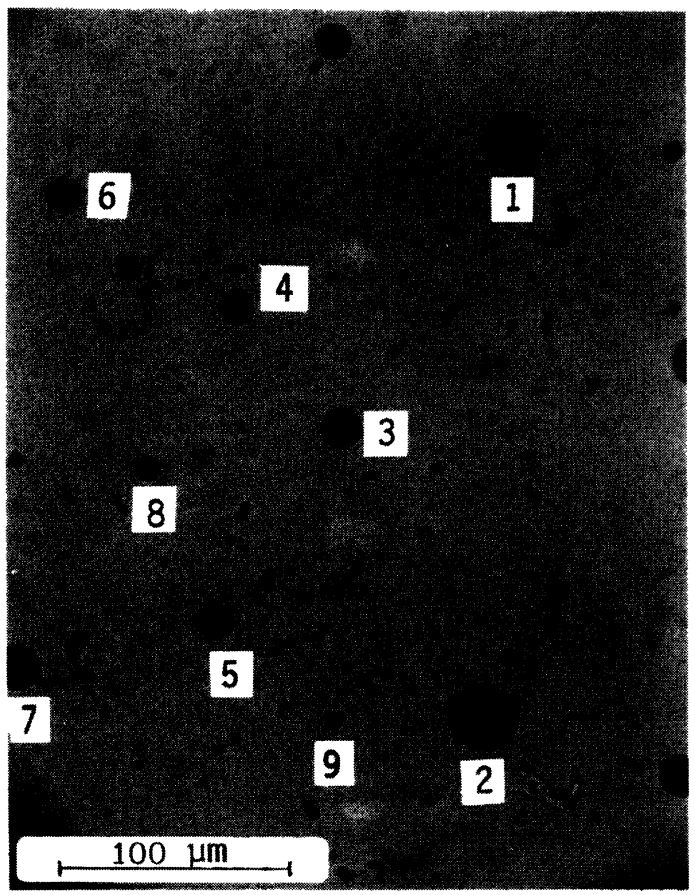

1)

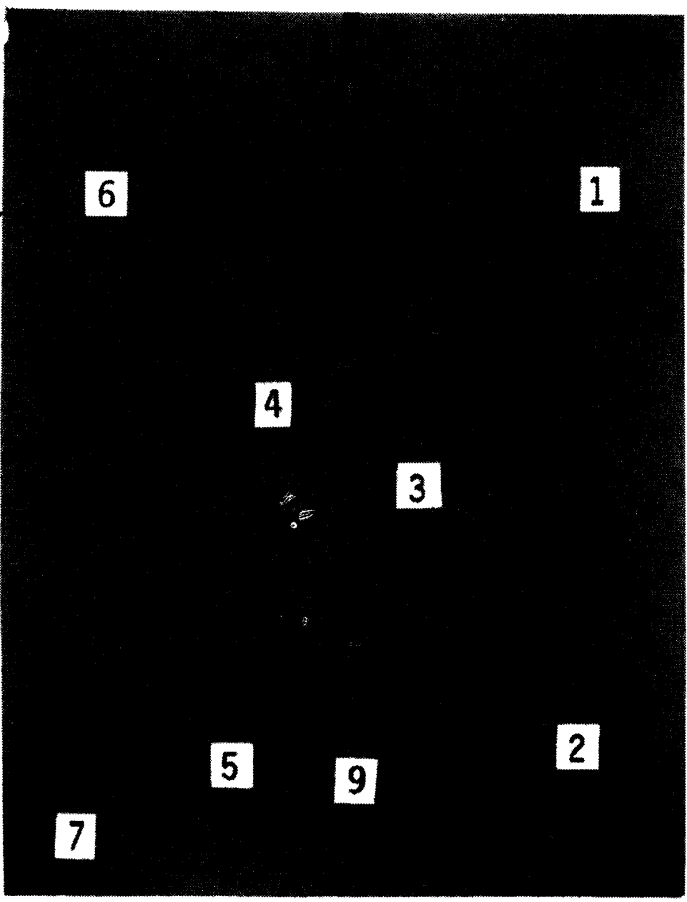

3)

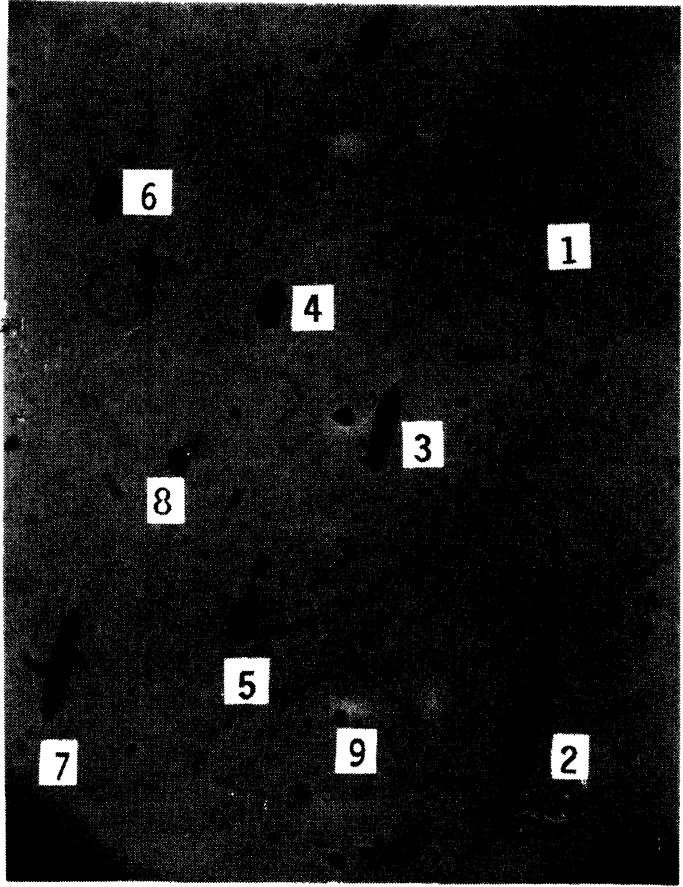

2)

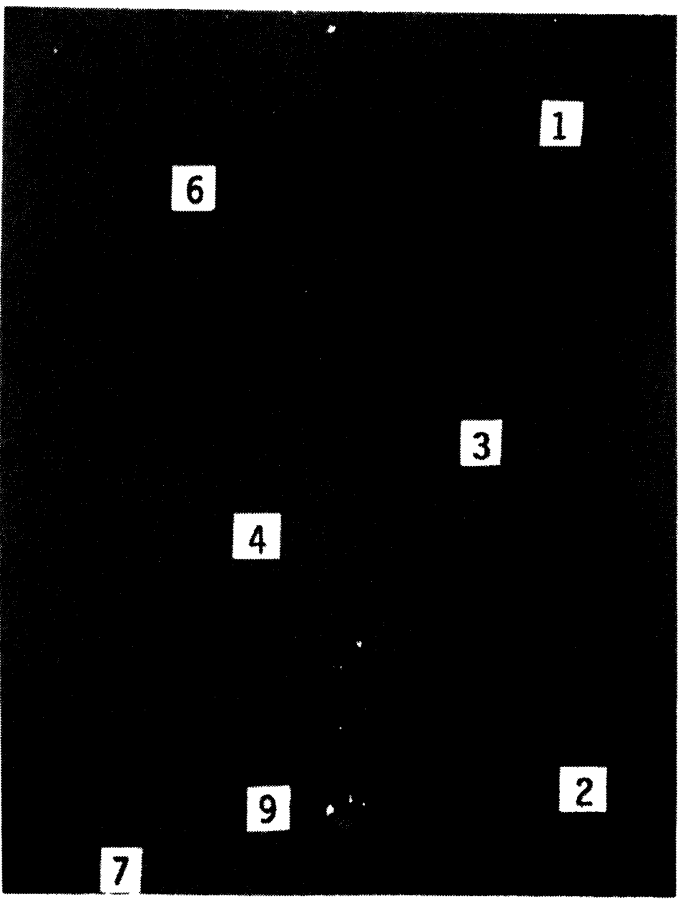

4)

Pictures. - Microscope pictures of the agglomerates for different magnetic fields : 1) $H=0$;2) $H=1 \mathrm{G}$; 3) $H=2.1 \mathrm{G}$; 4) $H=3.4 \mathrm{G}$. The field is in the plane of the picture and in the direction of the major axis of the ellipsoid. The scale is given in figure 1. 


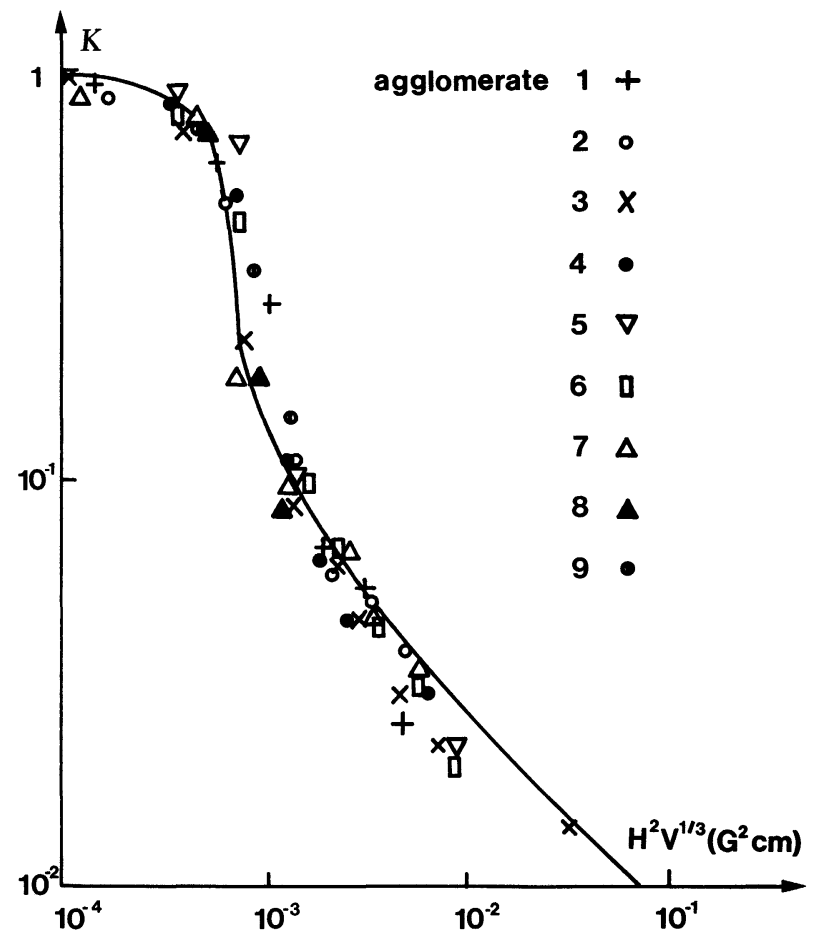

Fig. 1. - Log-Log plot of the aspect ratio $K$ versus the product of the square of the field $H$ by the cube roots of the volume $V$ of each agglomerate for each magnetic field. The nine different symbols correspond to the nine agglomerates of the pictures. The curve is the theoretical curve $f(K)$ with $Z=5$ (see the text).

two decades in $K$ for all sizes of agglomerates (three decades in $V$ ). Using our Log-Log plot, we can now try to fit this experimental universal function of $K$, independently of the value of $\sigma$, with the predicted function $f(K)$ (expression (7)). The only adjustable parameter is the value $Z(T)$, characterizing the exact shape of the cubic array of ferromagnetic dipoles in the agglomerate. The best fit is obtained for $Z(T)=5 \pm 0.1$ which is closed to the value at $T=0$ of a simple cubic lattice $\left(Z_{0}=5.352\right)$. The theoretical curve $f(K)$ drawn in figure 1 for $Z=5$ is in reasonable accord with our experimental data. This shows the validity of expressions (6), (7). The calibration between experimental and theoretical points gives the value of $\sigma$ :

$$
\sigma=(1.5 \pm 0.2) \cdot 10^{-4} \mathrm{erg} . \mathrm{cm}^{-2} .
$$

This value of the interfacial tension is two orders of magnitude larger than the value deduced by Liao and Krueger [13]. This difference can be explained by the difference between the two kinds of ferrofluid (with and without surfactant).

4. Conclusion. - We have measured the shape and size of large agglomerates of ferrofluid in a magnetic field. In order to interpret the data we adapt to our problem the work of Liao and Krueger on agglomerate formation in settling velocity measurements. Our simple model, based on a balance between magnetic energy and surface energy, quantitatively describes the deformation of ellipsoidal agglomerates in the magnetic field. Fitting to the data, with only one adjustable parameter, gives a value of the surface tension of the agglomerate in the ferrofluid suspension of $\sigma=(1.5 \pm 0.2) \cdot 10^{-4} \mathrm{erg} . \mathrm{cm}^{-2}$. 


\section{References}

[1] Rosensweig, R. E., Kaiser, R. and Miskolczy, G., J. Colloid Interface Sci. 29 (1969) 680.

[2] Kaiser, R. and Miskolczy, G., J. Appl. Phys. 41 (1970) 1064.

[3] Desai, J. N., Naik, Y. G., Mehta, R. V. and Dave, M. J., Indian J. Pure Appl. Phys. 7 (1969) 534.

[4] Roggwiller, P. and Kundig, W., Solid State Commun. 12 (1973) 901.

[5] Martinet, A., Rheol. Acta 13 (1974) 260.

[6] Hayes, C. G., J. Colloid Interface Sci. 52 (1975) 239.

[7] Hayes, C. F. and Hwang, S. R., J. Colloid Interface Sci. 60 (1977) 443.

[8] Hayes, C. F., Mol. Cryst. Liq. Cryst. 36 (1976) 245.

[9] Peterson, E. A., Krueger, D. A., Perry, M. and Jones, T. B., A.I.P. Conference Proc., Amer. Inst. Phys., N.Y. 24 (1975) 560.

[10] Peterson, E. A., Ph. D. Thesis, Colorado State University, Fort Collins, Colorado, U.S.A. (1975).

[11] Peterson, E. A. and Krueger, D. A., J. Colloid Interface Sci. 62 (1977) 24.

[12] De Gennes, P. G. and Pincus, P. A., Phys. Kondens. Mater. 11 (1970) 189.

[13] Liao, N. H., KRUeger, D. A., J. Colloid Interface Sci. 70 (1979) 564.

[14] Massart, R., C.R. Hebd. Séan. Acad. Sci. Paris 291 (1980).

[15] MASSART, R., I.E.E.E. Trans. on Magnetics 17 (1981) 1241.

[16] Luttinger, J. M. and Tisza, L., Phys. Rev. 70 (1946) 954.

[17] LANDAU, L. D. and Lifshitz, E. M., Electrodynamics of continuous medium (Pergamon Press).

[18] Jordan, P. C., Mol. Phys. 25 (1973) 962. 